\title{
Arthroplasty Treatment of Shoulder Arthritis
}

\section{Lynn A Crosby*}

Professor, Department of Orthopedic Surgery, Medical College of Georgia, Augusta, Georgia, USA

The first recognized shoulder arthroplasty was performed in 1893 but did not prove beneficial and was removed after a brief period for overwhelming sepsis. Dr. Charles Neer II is given credit for modern day arthroplasty success when he implanted his monoblock type of prosthesis in 1953 for the treatment of proximal humerus fractures. With this success in fracture treatment the use for arthritic conditions soon followed. Today orthopedic surgeons use shoulder replacement in the treatment of degenerative, rheumatoid, post traumatic, avascular necrosis, instability arthrosis, cuff tear arthropathy and other inflammatory arthritic conditions involving the shoulder joint.

The anatomic shoulder arthroplasty has for the last 30 years been the most common used shoulder prosthesis. This implant was either used only on the humeral side of the shoulder joint or hemi-arthroplasty or a total shoulder replacement which involved replacing both the humeral and glenoid with a prosthetic device. More recently the reverse total shoulder arthroplasty has become available to orthopedic surgeons in the United States and is quickly becoming the most popular arthroplasty used for shoulder replacement. This implant places the ball shaped component on the glenoid and the cup shaped component on the humeral side of the joint and thus is terms reverse prosthesis. This device was originally used in the United States in the late 1970's and early 1980's but failed to improve patients shoulder function or pain relief and was removed from use by the Federal Drug Administration.
This was mostly secondary to loosening problems associated with the inability to obtain fixation on the glenoid side. Over time the reverse prosthesis was improved with modern fixation techniques and it was rereleased into the United States Market in 2004. Currently there are many different designs available for treatment of arthritis with rotator cuff deficiency but all have the same basic concept. They use the deltoid muscle to stabilize the implant by bringing it out to its normal length and allowing the normal deltoid muscle action to elevate the shoulder joint as the prosthesis maintains stability.

In patients with intact rotator cuff muscles an anatomic shoulder replacement is still the prosthesis of choice and if the rotator cuff tendons should fail over time this implant can now be revised to a reverse prosthesis with only exchanging the glenoid side and the proximal portion of the humeral component. The humeral stem can be left in place in most cases as it is usually well fixed. This has made these revision procedures much less challenging for the surgeon but most importantly easier on the patient and less expensive.

These have been tremendous advances in the treatment of end stage arthritic conditions of the shoulder joint. There still is concern regarding long tern follow and what these outcome studies may reveal over time, however for the time being shoulder arthroplasty is providing excellent pain relief and functional return to an ever increasing number of people every year.
*Corresponding author: Lynn A Crosby, Professor, Department of Orthopedic Surgery, Medical College of Georgia, Augusta, Georgia, USA; E-mail: lycrosby@georgiahealth.edu

Received July 18, 2013; Accepted July 25, 2013; Published July 30, 2013

Citation: Crosby LA (2013) Arthroplasty Treatment of Shoulder Arthritis. J Arthritis 2: e109. doi:10.4172/2167-7921.1000e109

Copyright: (C) 2013 Crosby LA. This is an open-access article distributed under the terms of the Creative Commons Attribution License, which permits unrestricted use, distribution, and reproduction in any medium, provided the original author and source are credited. 